\title{
Voir, entendre et ressentir: à propos de l'écriture de Ce que j'appelle oubli de Laurent Mauvignier
}

\author{
Préparé par: \\ Dr. Dalia METAWE \\ Maître de conférences \\ Département de français \\ Faculté des lettres \\ Université de Menoufia
}





\section{Résumé}

Depuis ses publications, les œuvres de Laurent Mauvignier font toujours sensation. Elles se caractérisent par une originalité tant aux thèmes traités qu'à la pratique stylistique singulière de cet écrivain. L'écriture de Mauvignier est une écriture particulière qui donne à voir, à entendre et à ressentir. Elle accorde une place de première importance aux perceptions et aux sensations. Ce que j'appelle oubli est un roman de Mauvignier qui a été écrit en 2011 et a obtenu le prix de PACA en 2012. Ce roman se caractérise par un aspect multisensoriel qui se voit à travers un dispositif textuel riche en stylèmes visuels et sensoriels où certaines sensations tendent à assumer un rôle dominant dans le roman. Dans ce travail, nous étudierons les éléments visuels et sensoriels dans ce roman, tout en analysant leurs rôles dans l'organisation textuelle du roman. Nous examinerons comment Mauvignier a pu bâtir une esthétique de la perception basée sur le visuel et le sensoriel. Nous montrerons ensuite dans quelle mesure cet aspect multisensoriel pourrait, d'une part, offrir au lecteur une expérience affective particulière lui permettant de participer aux douleurs, aux déceptions et aux pertes des personnages du roman, et, d'autre part, comment il contribue à cerner la spécificité de ce roman.

$$
\begin{aligned}
& \text { إن الأعمال الأدبية للكاتب الفرنسي لورو موفينيه تثير المشاعر منذ نشرها حيث تتميز بأصالة }
\end{aligned}
$$

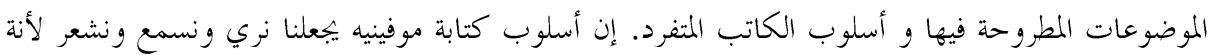

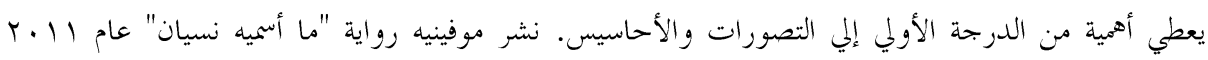

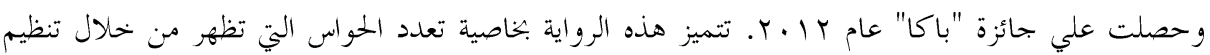

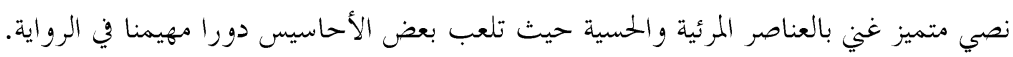

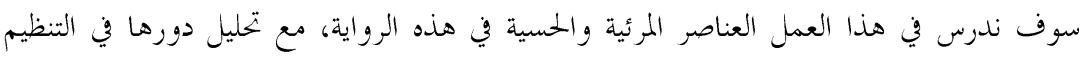

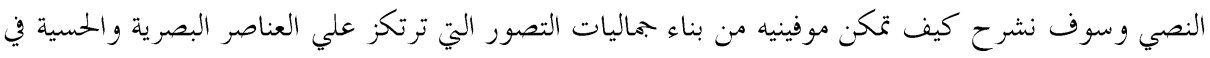

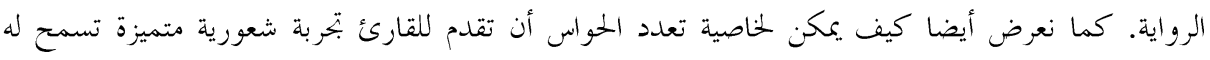

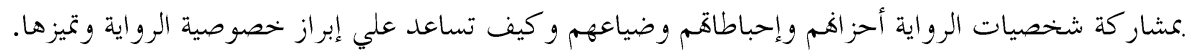


Laurent Mauvignier est un des écrivains français contemporains connu par la spécificité et la singularité de son style. La grande valeur de ses œuvres était le sujet de plusieurs articles parus dans les revues de presse et les journaux spécialisés ${ }^{1}$. Selon Jean-Claude Lebrun, le style remarquable de Mauvignier présente un exemple de "ce qu'on pourrait appeler un style" ${ }^{2}$. Christian Sauvage, pour sa part, parle d'un "miracle Mauvignier"3. . M. Bonazzi, de son côté, affirme bien justement que "le seul patronyme "Mauvignier" est devenu synonyme de la qualité stylistique- et donc par synecdoque, de la qualité romanesque-" ${ }^{4}$. L'écriture de Mauvignier est une écriture particulière qui donne à voir, à entendre et à ressentir. Elle accorde une place de première importance aux perceptions et aux sensations. Cette écriture explore les lieux de la sensation de façon qu'on ne sache plus si c'est la sensation qui oriente la langue ou bien c'est la langue qui infléchit la sensation pour bâtir un univers fictionnel dynamique plein de voix, de bruits, d'odeurs, de couleurs et de goût.

L'œuvre littéraire de Mauvignier, marquée par son succès public et critique, attire le lecteur sur deux plans: d'une part sa particularité thématique et significative, d'autre part, son côté non représentative (visualisation, rythme, sonorité). C'est sur ce deuxième aspect que nous nous concentrerons dans la présente recherche. Ce que j'appelle oubli est un roman de

${ }^{1}$ Dans son étude intitulée «"Ce qu'on pourrait appeler un style”: les représentations de la pratique stylistique de Laurent Mauvignier dans la critique littéraire » parue dans la langue de Laurent Mauvignier « une langue qui court », Mathilde Bonazzi regroupe, en annexe, un grand nombre de critiques parus dans la presse entre 1999 et 2009 à propos du style de Mauvignier. Cf., J. Dürrenmatt et C. Narjoux (dir.), Collection Langages, Éds. Universitaires de Dijon, 2012, p. 46-48.

2 Jean-Claude Lebrun, «Laurent Mauvignier, une insurmontable solitude », L'Humanité, 21/09/2000, en ligne: www.humanite.fr, consulté le 12/04/2017.

3 Christian Sauvage, «Le miracle Mauvignier », Le Journal du Dimanche, 3 avril 2005, en ligne: http://leseditionsminuit.fr, consulté le 15/04/2017.

${ }^{4}$ Matilde Bonazzi, op. cit., p. 17. 
Mauvignier qui a été écrit en 2011 et a obtenu le prix de PACA en 2012. Ce roman se caractérise par un aspect multisensoriel qui se voit à travers un dispositif textuel riche en stylèmes ${ }^{5}$ visuels et sensoriels où certaines sensations tendent à assumer un rôle dominant dans le roman. Pour encadrer notre travail, nous étudierons les éléments visuels et sensoriels dans ce roman, tout en mettant en relief les rôles qu'ils jouent dans son organisation textuelle. Nous examinerons comment Mauvignier a pu bâtir une esthétique de la perception basée sur le visuel et le sensoriel. Nous montrerons ensuite dans quelle mesure cet aspect multisensoriel pourrait, d'une part, offrir au lecteur une expérience affective particulière lui permettant de participer aux douleurs, aux déceptions et aux pertes des personnages du roman, et, d'autre part, comment il contribue à cerner la spécificité de ce roman.

Dans Ce que j'appelle oubli, Mauvignier fait un emploi très fréquent d'expressions relevant $d u$ vocabulaire visuel et sensoriel qui évoquent des effets de synesthésie. Le visuel constitue un aspect très marqué dans ce roman où nous repérons beaucoup de références liées au champ de la vue: lumière, couleurs. Nous examinons, à présent, cet aspect tout en analysant ses fonctions et ses valeurs dans la trame narrative du roman.

\section{1- Les sensations visuelles}

Inspiré d'un fait divers survenu à Lyon en 2009, Ce que j'appelle oubli est un roman court qui raconte l'histoire d'un homme tabassé à mort par quatre vigiles dans un supermarché à cause de la consommation, sur place, d'une canette de bière sans la payer. Ce roman est raconté par un narrateur anonyme omniprésent qui s'adresse au frère de la victime. L'œil joue un

${ }^{5}$ G. Molinié propose ce terme pour désigner les « caractérisèmes » de littérarité dans une œuvre. Cf. Georges Molinié, La stylistique, PUF, 2001, p.77-94, 203. 
rôle important dans ce roman dans la mesure où il voit, contemple le champ de la vue et conserve enfin les images captées dans le cerveau. La fixation sur le regard est un trait indispensable dans ce texte caractérisé par un emploi fréquent des verbes "voir" et "regarder". La victime, mais aussi le narrateur, ont une capacité de voir et d'observer de façon minutieuse, ce qui montre un désir acharné pour scruter le monde qui les entoure. Tout au long du roman, le narrateur voit et fait voir aux lecteurs, y compris le frère de la victime:

"[...] il [la victime] laisse flotter ses pas et ses pensées dans les allées où il regarde les carrelages blancs, les marques de roues des chariots, les traces de pas, les carreaux cassés et ceux qu'on a changés et qui sont plus clairs $[\ldots]^{\prime \prime 6}$.

La victime observe en faisant attention à tous les détails autour de lui, même les plus petits. Le monde diégétique est ainsi construit à partir de ses perceptions visuelles:

"[...] il voit qu'à leur ceinture tous portent une carte, un badge où est écrit sécurité, il y a leur photo et le nom qu'il essaie de lire mais très vite, au bout du rayon, le premier ouvre l'un des battants des grandes portes en plastique transparent avec les numéros dessus, en bleu, neuf et dix, issues de secours [...]"7

Ainsi le lecteur peut-il peindre une image précise de l'allure des vigiles à travers la description transmise par l'œil scrutateur et observateur du personnage-victime, ce qui produit un effet d'authenticité à la perception. Comme l'explique bien net Mauvignier lors d'un entretien avec Jean Laurenti que

${ }^{6}$ Laurent Mauvignier, Ce que j'appelle oubli (désormais Cqjo), Éditions de Minuit, 2011, p. 11. Dans cette citation ainsi que celles qui suivent, c'est nous qui mettons en italique. Nous commençons et terminons chaque citation par trois points entre deux crochets car ce roman ne contient ni majuscule, ni point.

${ }^{7}$ Ibid., p. 19-20. L'italique est de l'auteur. 
"C'est la perception des objets qui m'intéresse, pas les objets eux-mêmes" ${ }^{\text {. }}$. La modalité de perception constitue donc une des finalités de l'œuvre de Mauvignier pour élaborer un réalisme de l'intériorité ou plutôt de l'intime dans la mesure où il s'inscrit dans la lignée du roman réaliste français. Cependant le réalisme social et politique de Mauvignier est distingué par un regard original de la représentation du réel:

"Le but de l'écriture de Mauvignier n'est pas de reproduire du réel au sens descriptif du terme, comme Balzac par exemple, ni même la réaliste imitation d'un discours, comme Zola. Ce qui est reproduit, représenté par Mauvignier, c'est bien la perception intérieure du réel, avec tout ce qu'elle recèle de mouvant ; c'est-à-dire un réseau complexe de sensations, de surgissements de l'inconscient et de vagues introspectives du sujet [... ]"9 .

De ce point de vue, Yann Fayette, Remi Gonzalez et Anaïs Mateo qualifient Mauvignier d'écrivain impressionniste" $" 10$.

L'œil serait-il seulement l'organe de la vision qui permet au personnage-victime de regarder la scène autour de lui ? Il est vrai que les yeux de la victime percevaient son champ visuel, mais ils fonctionnaient également comme un miroir qui reflète ce qui se passe dans son intériorité et projette les sentiments qu'il ressent sans évoquer un mot:

8 «Capter la surface des choses», Entretien avec Jean Laurenti, Le Matricule des anges, Octobre 2006, en ligne: http://www.laurent-mauvignier.net/entretiens/dansla-foule/capter-la-surface-des-choses.html, consulté le 5-7-2017.

9 Yann Fayette, Remi Gonzalez et Anaïs Mateo, « Mauvignier: impressions », La Langue de Mauvignier: « une langue qui court», op. cit., p. 51.

${ }^{10}$ Loc. cit. 
"[...] ils l'ont encerclé et il n'a pas eu un mot pour contester ou nier [...], il n'a pas dit un mot et dans ses yeux il a laissé le jeu ouvert de la peur [... $]^{\text {"11 }}$.

La frayeur ressentie de ce pauvre homme face à une situation grave à venir apparaît dans ses yeux. Cependant, ce n'est pas le seul rôle des yeux car, en dévoilant ce qui se passe dans l'intériorité de la victime, il lui révèle, en même temps, ce qui se passe dans la conscience des autres, en l'occurrence les quatre vigiles du supermarché, comme le témoigne l'exemple suivant:

"[...] il sait qu'ils vont lui casser la gueule, parce qu'il le voit de la façon qu'ils ont de s'envoyer des coups d'cil entre eux pour se motiver, ils s'amusent, ils font semblant de se mettre en colère" ${ }^{912},{ }^{6}[. .$.$] , il ne sait pas$ dire ce qu'il voit encore dans leurs regards, ce sérieux, cette application comme si leur vie en dépendait, $[\ldots]^{9913}$.

Ici, l'action de voir n'est pas une forme de voyance simple, elle prend également le sens de comprendre. Dans ce cas, les coups d'œil envoyés entre les quatre vigiles sont révélateurs de ce qu'ils ont l'intention de faire. Cette forme de voyance donne lieu à la compréhension de la victime de la résolution prise par les quatre vigiles de lui "casser la gueule", expression répétée plusieurs fois par la victime ; autrement dit, la capacité de voir- au sens de comprendre- que le jeune homme possède lui permet de révéler le fonctionnement psychique dans la conscience la plus intime des vigiles. L'action de voir constitue donc un élément générateur de l'événement traumatique du roman.

${ }^{11}$ L. Mauvignier, Cqjo, op. cit., p. 8.

12 Ibid., p. 21.

${ }^{13}$ Ibid., p. 26. 
Ajoutons que l'action de voir devient une des activités quotidiennes de la victime; même aux moments difficiles quand il se sent découragé et ne voulant rien faire, il ne cesse jamais de regarder et de contempler le monde autour de lui. Dans ce cas, la capacité de voir de l'homme marginal se transforme en une force de regarder:

"[...] il n'avait que la force de regarder les pigeons et les moineaux qui volent dans la gare et se posent sur les blocs de béton, au dessus des gens, des valises et des sacs, et c'est comme dans les cathédrales, avec les moineaux et les pigeons qui roucoulent, enfermés on dirait, sous les charpentes, comme les tourterelles aussi dans les granges, ça rappelle l'été, le printemps [.... $]^{\text {14 }}$.

Dans cet exemple, le narrateur du roman voit et nous fait voir, en focalisation interne, ce que le jeune homme voit dans son errance. Les événements sont ainsi perçus à travers les regards du narrateur omniscient qui a une vision d'ensemble de l'espace et du temps. Dans cette perspective, nous pensons qu'il serait très intéressant de détecter la direction des regards du narrateur qui indique en même temps la position de son corps dans la mesure où l'acte de voir du narrateur ne montre pas seulement le geste de son œil, mais il désigne également la position de son corps par rapport à celui de la victime. Pour ce faire, nous avons en effet trois possibilités:

- le narrateur se trouvait à droite ou à gauche de la victime ;

- le narrateur regardait à peu près la victime en face ;

- le narrateur regardait tout d'en haut.

Le narrateur de ce roman est omniprésent voyant tout et connaissant tout de son personnage: ses pensées, son passé et

${ }^{14}$ Ibid., p. 47. 
son avenir. Pour pouvoir accéder directement et largement aux scènes, sans rencontrer aucun obstacle ni empêchement, le narrateur doit se placer au dessus de tout, ressemblant en cela à la caméra cinématographique qu'on place au dessus de lieu de tournage pour tout filmer. Telle position supérieure permet au narrateur d'acquérir une vue panoramique de l'univers romanesque présenté avec tous les détailles possibles. Ainsi, l'œil du narrateur regarde-t-il le plus souvent la scène d'en haut pour tout voir. Il s'agit donc d'une vue située au-dessus de tout, comme le montre le schéma suivant:

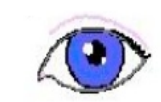

\section{L'œil (le corps) du narrateur qui voit tout d'en haut}

La scène décrite

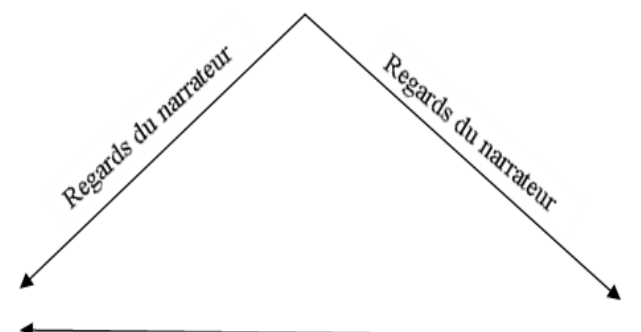

Regards du jeune homme

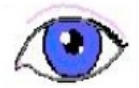

L'œil de la victime

À côté de l'œil du narrateur qui voit et fait voir au lecteur, il existe également un dispositif cinématographique, à savoir les caméras de surveillance qui montrent l'intérêt que porte Mauvignier au cinéma:

"[...], les caméras au-dessus de lui le regardent entre les annonces des sorties de secours et les promos, au moment de les voir arriver il pense que son programme de la journée est compromis [.... $]^{\text {"15 }}$.

Notons que l'existence des caméras qui enregistrent ce qui se passe dans le supermarché implique une visée intentionnelle que nous trouvons également à l'image de la

${ }^{15}$ Ibid., p. 17. 
vierge phosphorescente qui, placée au-dessus de l'armoire, contemple la victime et son frère toutes les nuits avec quiétude et calme, dans leur chambre à coucher. Les yeux de la vierge phosphorescente se présentent comme une caméra de surveillance provoquant la peur et l'inquiétude chez la victime pendant son enfance tout en l'empêchant de dormir:

"[...] et les peurs d'enfant qu'il [la victime] avait en regardant au dessus de l'armoire, face au lit, la vierge phosphorescentes dans sa boule de verre et la neige, ce qui l'empêchait de dormir et le laissait fixe dans le noir comme un hibou effaré sur une branche, alors qu'il voulait dormir, il veut dormir, il va dormir enfin et ce sera comme quand les mains sur les corps disparaissent et laissent place au silence, $[\ldots]^{\text {"16 }}$.

Le goût de Mauvignier pour le cinéma se voit également à travers les multiples références aux films qu'il cite dans le roman, comme le témoignent les exemples suivants:

"[...], il n'a vu ça que dans les films et ces corps aussi, dans les films, avec les blessures déjà froides que le médecin légiste et la police regardent d'un oil détaché avant qu'on rabatte sur le visage un tissu blanc, un plastique, [... $]^{9,17}$, «[...] il ne savait pas qu'il mourait, dans les films ils savent toujours qu'ils meurent, mais en vrai ce n'est pas aussi beau, on n'est pas si beau, [... $]^{\text {"'18 }}$

Dans Ce que j'appelle oubli, Mauvignier fait plusieurs fois référence aux films pour, à notre avis, montrer la réalité choquante et cruelle dans notre vie quotidienne. Cet emploi

${ }^{16}$ Ibid., p. 53.

${ }^{17}$ Ibid., p. 18.

${ }^{18}$ Ibid., p. 34

عدد 01- ربيع م| • 
met essentiellement en relief la dimension visuelle de ce texte. Remarquons que le recours au cinéma est fréquent chez les romanciers contemporains comme, entre autres, Éric Laurrent et Tanguy Viel, en vue de donner beaucoup de visualité à la création artistique contemporaine. Elisa Bricco affirme justement qu' 'on ne peut qu'être d'accord que le cinéma fait partie de notre encyclopédie culturelle et que l'on ne se réfère plus seulement à cet art en fonction des thématiques et des techniques partagées, mais les auteurs ont intégré le mouvement cinématographique ainsi que les manières de s'approcher de la vision des films dans leurs ouvrages (voir Cinéma de Tanguy Viel et aussi Pas le bon pas le truand de Patrick Chatelier)" "19. Elle ajoute que "Le cinéma devient un thème au sens fort du terme, à même d'imprégner l'écriture et la structure des ouvrages voire le langage utilisé. Ainsi fonctionne aussi le rapport du texte avec la photographie" ${ }^{20}$. Ainsi s'exprime Fusillo: «L'auteur ne vise donc pas à décrire et/ou à raconter l'expérience autre de la vision cinématographique, mais à imprégner l'écriture de mémoire filmique et visuelle, souvent par allusions ou par le biais de citations directes, et souvent impliquant la vie et le regard des personnages, surtout à propos d'icônes désormais inscrites dans l'imaginaire contemporain comme Greta Garbo..l ${ }^{21}$

De ce qui précède, nous constatons que la vue présente le sens dominant dans ce texte où les perceptions visuelles

${ }^{19}$ Elisa Bricco, « Notes à partir de L'esthétique de la littérature entre performativité et visualité de M. Fusillo », ARGEC, Atelier de recherche génois sur les écritures contemporaines, 15/06/2012, en ligne,http://argec.hypotheses.org/243, consulté le10/07/2017. Pour plus d'information sur la relation entre la littérature et le cinéma, voir Jean-Louis Leutrat (dir.), Cinéma et littérature - le grand jeu, De l'incidence (éditeur), 2010; Jeanne-Marie Clerc, Littérature et cinéma, Coll. Fac. Cinéma, Nathan Université, 1999.

${ }^{20}$ Loc.cit.

${ }^{21}$ Cité par Elisa Bricco, Ibid. 
constituent une pratique majeure dans la vie quotidienne du personnage. Cette importance donnée au visuel est due, nous semble-t-il, à l'art cinématographique qui exerce, de plus en plus, une grande influence sur les écrivains postmodernes en modifiant la modalité de la représentation de leurs œuvres. Selon cette perspective, nous remarquons que Mauvignier, pour sa part, intègre dans son texte des techniques cinématographiques plus axées sur le visuel pour mieux inclure le lecteur dans la scène en le plaçant directement comme spectateur. Pour cet écrivain, le cinéma:

"[...] est un moteur d'écriture: il concurrence le réel comme le roman a pu le faire il y a longtemps, et il est un réservoir d'idées, de formes. Bien évidemment, il infléchit notre travail sur les questions de mises en avant de détails, il nous interpelle sur le montage, le découpage, le cadrage, sur la question du temps et du récit, du scénario bien sûr, mais aussi sur des plans autres que narratifs ou formels ${ }^{\text {"22 }}$.

L'influence du cinéma sur la pratique stylistique de Mauvignier se voit également à travers la présence de descriptions détaillées et minutieuses fondées sur un ensemble de termes visuel et sensoriel particulier qui caractérise Ce que j'appelle oubli, comme le montre l'exemple suivant:

${ }^{6}[. .$.$] et devant eux des jeunes passent en rollers avec$ plusieurs paniers vides dans chaque main, et l'odeur de poisson, le froid des surgelés et les jambons sous

22 Johan Faerber, Entretiens, Laurent Mauvignier, Livres, Rentrée littéraire 2016, le 1 septembre 2016, en ligne: https://diacritik.com/2016/09/01/laurent-mauvignier-il-y-a-deslivres-qui-veulent-nous-soumettre-a-nos-peurs-plutot-que-de-les-interroger/, consulté le $17 / 12 / 2017$ 
vides, il a le temps de voir, de tout voir, la déco des stands, la boulangerie qui la joue rétro façon parisienne et la fromagerie qui veut ressembler à une ferme, $[\ldots]{ }^{23}$.

Le personnage éprouve une volonté de tout voir. La diversité des détails enrichit constamment le champ visuel de la victime souvent composé d'une multitude d'images. Dans ce cas, l'acte de voir contribue à dessiner une image saturée d'objets où les détails construisent la scène toute entière. Notons que la portée ironique de cette description est évidente ${ }^{24}$. Elle apparaît clairement à plusieurs reprises dans le roman dont nous citons par exemple, dans le centre commercial, le "faux mur végétal" 25 , la "pelouse synthétique",26" "les couleurs criardes des promos" 27 et la blonde "décolorée et

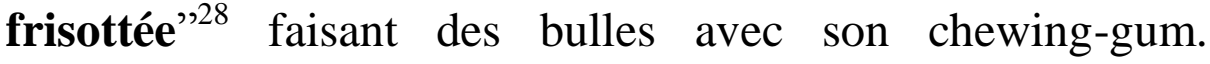
L'ironie s'exerce également contre les vigiles, perceptible dans leur description:

"[...] le costume mal taillé et la boule à zéro du plus jeune des quatre, que celui-ci doit raser tous les jours pour se donner l'air mauvais ou crédible $[. . .]^{929},{ }^{6}[\ldots]$ le plus jeune, celui qui se rase le crâne bien net pour faire vigile [.... ", 30

C'est comme l'affirme H. Gustafsson que: "la technique d'utiliser le visuel nous permet de voir ce qui ne peut pas toujours être dit. Mauvignier a un sens très

${ }^{23}$ L. Mauvignier, Cqjo, op. cit., p. 19.

${ }^{24}$ Voir sur ce sujet l'article de Karine Germoni, «L'ironie façon Mauvignier », Existe-t-il un style Minuit ?, M. Bertrand, K. Germoni, A. Jauer (dir.), Aix-enProvence, PUP, 2014.

${ }^{25}$ L. Mauvignier, Cqjo, op. cit., p. 10

${ }^{26}$ Loc. cit.

${ }^{27}$ Ibid., p. 11

${ }^{28}$ Loc. cit.

${ }^{29}$ Ibid., p. 12 et 13

${ }^{30}$ Ibid., p. 24. 
poussé du détail qui donne des images et des sensations immédiates chez le lecteur. C'est par ces images que surgit la signification" ${ }^{31}$.

Ainsi, la description permettant la révélation possède une puissance poétique du faire-voir et constitue un des éléments dans le roman qui visualise. La description donne également à comprendre sans évoquer le mot exact, comme dans l'exemple suivant:

"[...] ou les clients de votre père, tu les vois ? tu les entends ? donnez-moi deux beaux steaks pas trop épais et puis, avant de partir, hésitant un peu, faussement timides, au lieu de dire comme d'habitude, il fait beau aujourd'hui, prendre la mine de circonstance, on a appris pour votre fils, dans quel monde on vit, hein, allez, bon courage, tu les entends? tu entends ça ? [...]" ${ }^{\prime 32}$.

Nous comprenons alors que le père de la victime est boucher, et cela sans le révéler directement mais grâce aux détails descriptifs qui guident le lecteur dans son processus de lecture et d'appréhension. Grâce à cette stratégie particulière basée sur l'emploi de multiples éléments détaillés visuels et sensoriels, le lecteur peut restituer la scène et comprendre ce qui se passe avec clarté et précision. Mauvignier révèle son goût pour les détails en disant que: "les détails permettent de dire beaucoup de choses qui ne passeraient pas sans eux" ${ }^{33}$.

${ }^{31}$ Harriet Gustafsson, L'œuvre Romanesque de Laurent Mauvignier, Mémoire sous la direction du professeur Björn Larsson, Université de Lund, Centre de langues et de littérature, 2012 , en ligne: lup.lub.lu.se/studentpapers/record/3812729/file/3812804.pdf, consulté le 29/3/2017.

${ }^{32}$ L. Mauvignier, Cqjo, op. cit., p. 33.

${ }^{33}$ " Misère de l'amour. Entretien avec Jean Laurenti », Le Matricule des Anges, mars 2004. En ligne, http://www.laurent-mauvignier.net/entretiens/seuls/misere-de-1amour.html. Consulté le 24/05/2017. 
L'emploi abondant des couleurs constitue un autre aspect dans le roman qui visualise. Les trois couleurs primaires le rouge, le bleu et le jaune sont fréquemment présentes, ainsi que les couleurs secondaires comme le vert, le gris et le noir: "fenêtre blanche" 34 "Sa peau toute blanche [...] ses yeux verts" $" 35$ " "tee-shirt jaune et noir" "les mots noir sur blanc" "37, "les lèvres bleues"38, ou encore "le bleu du ciel, le gris de l'eau"39.

Nous repérons également un vocabulaire insistant sur la qualité de la couleur comme "sang rouge vif" "couleurs criardes" $"$. D'autre part, l'auteur fournit parfois des précisions utiles de l'ordre du visible comme par exemple "cheveux couleur de paille"42 et "noir de cendre" L'abondance des couleurs, surtout le blanc et le noir évoqués plusieurs fois dans le roman, met la dimension visuelle et sensorielle du roman en relief dans la mesure où ces couleurs véhiculant des sensations mélancoliques ayant des rapports avec la mort, le deuil, le froid, l'indifférence et l'absence de sentiments, visualisent.

Ajoutons que la volonté de faire voir s'affirme par l'emploi de la lumière qui domine dans le roman de façon passionnante:

"[...] un jour comme aujourd'hui où la lumière était blanche comme une lame de couteau brillant sous un néon dans une cuisine- il s'est souvenu du papier

\footnotetext{
${ }^{34}$ L. Mauvignier, Cqjo, op. cit., p. 9

35 Ibid., p. 23

${ }^{36}$ Ibid., p. 31.

${ }^{37}$ Ibid., p. 32

${ }^{38}$ Ibid., p. 42.

${ }^{39}$ Ibid., p. 57.

${ }^{40}$ Ibid., p. 30.

${ }^{41}$ Ibid., p. 11.

42 Ibid., p. 12.

${ }^{43}$ Ibid., p. 42.
} 
peint avec les cerises rouges et de comment elles éclataient dans la nuit, à cette fenêtre blanche et au néon si blanc et vibrant lui-aussi, quand il rentrait chez lui à sept heures du matin après avoir baisé sur les bords de la Loire» ${ }^{44}$.

Dans cet exemple il existe un emploi abondant de la lumière blanche pour accentuer l'effet du jour et de la clarté. Remarquons que la brillance, se manifestant à plusieurs reprises dans le roman, constitue un autre élément qui donne à voir avec éclat, comme dans ces exemples: "le gel qui brille"45, "le film plastique brille" ${ }^{46}$. Cependant, la lumière pâle est également présente pour nuancer la sensation de la lumière dont nous repérons:

"[...] son visage lisse et bleu sous cette lumière pâle comme le reflet d'un tube de néon sur une lame de couteau [.... $]^{\text {"947 }}$.

Dans cet exemple, la représentation de la lumière se concrétise par l'image du cadavre de la victime.

Comme nous avons montré plus haut, Ce que j'appelle oubli se caractérise par l'abondance des signes visuels, ce qui met en relief la visualité du roman. Ajoutons que, sur le plan lexical, Mauvignier emploie fréquemment le pronom démonstratif indéfini "ça" en vue de "désigner l'incapacité des personnages à s'exprimer sur leurs expériences traumatiques ${ }^{48}$. Ce pronom, malgré son statut indéfini, constitue un des dispositifs dans le roman qui visualise. Citons cet extrait situé à la fin de l'œuvre:

${ }^{44}$ Ibid., p. 8-9.

${ }^{45}$ Ibid., p. 13.

${ }^{46}$ Ibid., p. 20

${ }^{47}$ Ibid., p. 42.

48 Myrthe Holvoet, Laurent Mauvignier et le minimalisme: l'écriture du non-dit, Mémoire de maîtrise sous la direction de Pierre Schoentjes, Université Gent, 20122013, p. 33. 
"[...] alors je ne me plains de rien sauf d'avoir glissé trop vite, si vite, dans la mort, de ne pas avoir su résister un peu, mais, je te l'ai dit, toujours cette connerie d'espoir qui me fait croire que ça va s'arranger, ça va aller, qu'est-ce que tu en penses ? tu ne crois pas que si les gens voulaient ça vaudrait le coup d'attendre le plus longtemps possible de ce côté-là de la vie ? mais ça, c'est encore une façon d'espérer un truc, comme au dernier moment, quand il y avait cette voix qui continuait et répétait, pas maintenant, pas comme ça, jusqu'à ce qu'elle se taise elle aussi et s'efface dans un chuchotement, trois fois rien, un sifflement, sa voix à lui qui continuera dans ta tête, à murmurer, à répéter toujours pas maintenant, pas maintenant, pas comme ça, pas maintenant-" ${ }^{49}$.

L'emploi abondant de "ça" visualise au lecteur la mise à mort de la victime par les quatre vigiles. M. Holvoet signale que "le narrateur éprouve des difficultés à exprimer les événements traumatiques par des mots concrets" ${ }^{\text {"50 }}$. Il ajoute que cette impuissance de s'exprimer, symbolisée par l'emploi du pronom indéfini "ça", vide de sens réel, forme un exemple du phénomène du non-dit dans les œuvres de Mauvignier ${ }^{51}$. Cependant, malgré cette difficulté à dire par des mots concrets, le "ça" matérialise l'innommable en imposant brutalement l'image du meurtre commis par les quatre vigiles devant les yeux du lecteur.

Il est à remarquer que Mauvignier met en œuvre dans ce roman d'autres expressions qui ont le même référent dont nous citons par exemple: “[...] et tant pis s'ils n'ont rien

${ }^{49}$ L. Mauvignier, Cqjo, op. cit., p. 61-62.

${ }^{50}$ Myrthe Holvoet, op. cit., p. 31.

${ }^{51}$ Loc.cit. Voir également p. 52-56. 
entendu quand le procureur a dit qu'on ne tue pas un homme pour ça, $[. . .]^{952}$, "[...], on ne tue pas un homme pour une chose comme celle-là, [...]" ${ }^{933}$, ou encore: "6[...] c'est qu'un homme ne doit pas mourir pour si peu [... $]^{9954}$. Toutes ces variantes concrétisent la scène du meurtre de la victime en la faisant voir au lecteur.

Comme nous l'avons montré, le visuel joue un rôle majeur dans ce roman. Il présente un tableau riche de stylèmes relatif à la vue que ce soient des verbes, des adjectifs ou bien des noms, avec beaucoup de lumières et de couleur. Ce qui est, en effet, le plus fascinant dans ce roman, c'est la façon de montrer visuellement ce qui se passe au lieu de tout dire. H. Gustafsson explique que l'originalité de ce roman se voit dans: "la façon que [Mauvignier] utilise le monologue intérieur; comment il maîtrise le visuel entre autres par le soin des détails dans les images, par le soin de choix des mots, par le rythme et la musicalité dans la langue. Sa capacité d'éviter le mièvre et d'omettre des jugements personnels confère à mon avis une grande valeur à son œuvre" ${ }^{95}$.

Mauvignier élabore ainsi une esthétique basée sur la visualité qui constitue un élément fondamental dans la matérialité textuelle de ce roman. Il s'agit d'un choix intentionnel de l'auteur qui explique:

“je visualise très bien les scènes, j’écris jusqu'à ce que je retrouve par la langue cette densité visuelle. Mais très vite, il y a un moment où l'écriture prend le pas, s'autonomise, elle embarque le visuel avec le sonore, la sensation, dans le rythme de la phrase.

${ }^{52}$ L. Mauvignier, Cqjo, op. cit., p. 32.

${ }^{53}$ Ibid., p. 37.

${ }^{54}$ Ibid., p. 7.

${ }^{55}$ Harriet Gustafsson, op.cit, p. 26-27. 


\section{Le phrasé, finalement, produit toujours davantage} d'images, de sons, etc., que je ne saurais en imaginer" $"$.

Si les sensations visuelles jouent un rôle très important dans le roman, les sensations non-visuelles n'y sont pas absentes. Elles occupent également une place dominante dans ce texte serré et visuel. Nous étudions, à présent, leur fonctionnement et leur valeur dans le roman.

\section{Les sensations non-visuelles}

Les sensations non-visuelles jouent un rôle important dans ce roman dans la mesure où elles créent un ton affectif qui submerge le lecteur et lui fait revivre les événements tragiques du roman. Les sensations non-visuelles se divisent en quatre genres correspondant aux quatre sens suivants: l'ouïe, l'odorat, le goût et le toucher. Nous avons montré plus haut comment la vue joue un rôle dominant dans le roman, nous mettrons, à présent, l'accent sur les autres sens.

\subsection{Les sensations auditives}

Ce que j'appelle oubli, roman constitué d'une seule phrase étendue sur soixante-deux pages, est débordé par des voix, des sons et des bruits qu'on peut entendre tout au long du roman. Remarquons que l'action de voir et d'entendre établit une relation d'équivalence où la vue stimule constamment l'ouïe dans une activité réciproque interminable dont voici un exemple:

\section{"[...] les gens viennent entre amis ou en famille et un chewing-gum éclate dans la bouche d'une blonde décolorée et frisottée, juste avant la rangée des caisses}

56 Françoise Dubor, «Entretien avec Laurent Mauvignier autour de Dans la foule (2006)». Cahiers Forell. En ligne: http://09.edel.univpoitiers.fr/lescahiersforell/index.php?id=251, consulté le 27/07/2017. 
où on entend les bips des articles sous la douchette des caissières, et il va sur la droite, vers l'entré, et bientôt dans le magasin il marche dans les rayons en se laissant porter par le son métallique des chansons à la radio et les couleurs criardes des promos [... $]^{957}$.

Le narrateur décrit le moment où la victime entre dans le supermarché, endroit du meurtre, tout en montrant les sons et les bruits qui circulent dans le magasin. Dans cette scène, nous distinguons plusieurs sources sonores: les voix des gens qui parlent, le son d'un chewing-gum qui éclat dans la bouche d'une blonde, les bips des machines à la caisse, les chansons à la radio. Le lecteur, grâce à ce style, vit la scène en la voyant et en l'entendant avec beaucoup de vivacité et de dynamisme.

Prenons un autre exemple qui montre clairement la complémentarité entre la perception visuelle et celle auditive affirmée par la fréquence de l'emploi des verbes "voir" et "entendre":

"[...] ce n'est pas comme le vide ni non plus le néant, pas la mort, pas le rien, c'est comme un rocking-chair avec l'osier qui craque en balançant, c'est calme et doux comme de voir les étoiles un soir d'été et d'entendre les grenouilles du ruisseau d'à côté, c'est comme la fermeture Éclaire de la toile de tente$[\ldots]^{9,58}$.

Les sensations auditives se manifestent donc par l'utilisation récurrente des verbes "entendre" et "écouter", mais aussi par le choix d'un vocabulaire laissant entendre une multitude de sons et de bruits divers dont nous citons à titre d'exemple:

${ }^{57}$ L. Mauvignier, Cqjo, op. cit., p. 11.

${ }^{58}$ Ibid., p. 56. 
"[...] une main le gifle [...], ça résonne dans sa tête comme le son d'une sirène qui sifflerait trop près et trop fort [...]" "s9, "le nez éclate"60, "le foie explosé" "combien de coups pour ne plus entendre son corps se froisser comme une canette écrasée sous les doigts ?"62.

Les mots en italique nous donnent à entendre des sons criards et hauts. Notons que la perception auditive se fait à travers l'oreille du narrateur qui se trouve, nous semble-t-il, en position supérieure par rapport aux personnages comme le montre le schéma suivant:
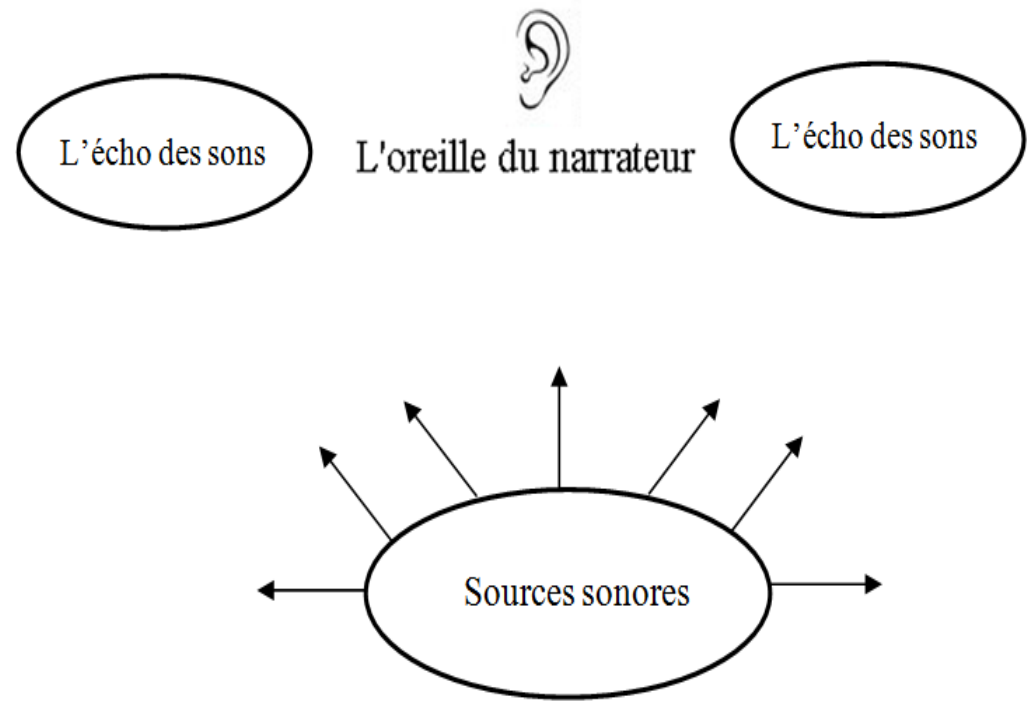

Cette position supérieure permet au narrateur de tout entendre (sons et écho) avec clarté et netteté.

Par ailleurs, la fréquence et la multiplicité des sons et des bruits qui caractérisent l'univers fictif de ce roman sont parfois

\footnotetext{
${ }^{59}$ Ibid., p. 21.

${ }^{60}$ Loc. cit.

${ }^{61}$ Ibid., p. 18.

${ }^{62}$ Ibid., p. 52.
} 
entrecoupées par des moments de silence. Cependant, le lecteur pourrait entendre, même aux moments de silence, des bruits paradoxaux qui surgissent pour mettre en évidence la lourdeur du silence s3 $^{3}$ et la difficulté à s'exprimer. Ces bruits provoquent chez le lecteur une certaine tension avec beaucoup de malaise et d'inquiétude, comme dans l'exemple suivant représentant le frère de la victime qui reconnaissait son corps mort et signait le papier de police:

"[...] c'est sur en voyant son corps, en disant, c'est lui, oui, c'est mon frère, et tu as reconnu ton frère mais tu n'as pas reconnu ta voix disant c'est mon frère, c'est lui, c'est mon frère, signez ici, et toi tu hochais la tête en entendant le stylo sur le papier"64.

$\mathrm{Au}$ fond du silence, le stylo fait un bruit en signant le papier. Le lecteur pourrait entendre, de même, dans le vide dépourvu de matières et de gens, l'écho du son, comme dans l'exemple suivant qui marque l'isolement de l'endroit où les quatre vigiles emmènent la victime pour lui donner des coups de pied et des coups de poigne de plus en plus fort et de plus en plus vite:

"[...] tous les quatre étaient là pour regarder sa bouche ouverte sur la dalle de béton où le sang grandissait et s'étalait- et pourtant, avant ça il a résisté un peu, ils le frappent sans se parler et le bruit que ça fait dans son corps résonne sous les tôles du plafond, très haut, l'écho dans les réserves, leurs souffles et son souffle, les râles qui se répercutent au loin comme des balles de tennis,

${ }^{63}$ Voir sur ce sujet l'étude de Aude Laferrière, «Entre «le silence et l'écho », les réticences et les réminiscences verbales: L'écriture des discours rapportés dans Loin d'eux et Des hommes », La langue de Laurent Mauvignier: « une langue qui court », op. cit., p. 61-79.

${ }^{64}$ L. Mauvignier, Cqjo, op. cit., p. 55 
parfois le bruit d'un chariot qui charge des palettes et eux, de le voir contre le mur de conserves, apeuré, l'air fou bientôt, ça les excite encore, sans qu'ils le disent $[. . .]^{\text {"965 }}$.

Cet extrait montre le processus du meurtre que les quatre vigiles ont commis et l'extrême violence qu'ils ont exercé contre la pauvre victime. C'est une scène pénible, marquée par la pluralité de bruits et d'écho qui résonnent haut et fort au fond dans les réserves et de façon répétitive entremêlant le bruit d'un chariot, le bruit des coups des vigiles, leur souffle, le souffle de la victime et ses râles. Cette scène provoque le sentiment de tension chez le lecteur qui a l'impression de voir le crime et d'entendre les bruits des coups et les agonies de la victime qui était sur le point de mourir. La comparaison entre le bruit de la scène du crime et celui des balles de tennis renforce l'idée de la violence et la répétitivité des coups des vigiles qui était l'objet de plusieurs autres reprises dont nous citons à titre d'exemple:

"[...] il n'a pas crié ou alors au début, si peu, il s'est débattu mais ce n'était presque rien, il a mis ses mains devant son visage et ils ont giflé, giflé encore et les coups pleuvaient qu'il entendait dans sa tête s'amplifiant comme des vagues, et puis, ils ont frappé le ventre et les jambes, [... ["66.

Pendant cette scène pénible du meurtre, le lecteur pourrait, grâce à la spécificité du style de ce roman, ressentir et entendre les souffles des vigiles ainsi que les souffles de la victime incapable de dire un mot:

"[...] quand il essaie de donner des coups de pied devant lui, alors ils le lâchent et il tombe dans un

${ }^{65}$ Ibid., p. 25.

${ }^{66}$ Ibid., p. 58. 
grand bruit de souffle coupé [...]" "67, "le jeune mec avec sa gorge sèche respire comme s'il avait mal" ${ }^{\prime 68}$.

Notons que le souffle établit un rapport avec le nondit $^{69}$ car, comme l'explique Myrthe Holvoet, "d'une part il évoque le silence et d'autre part, il est nécessaire de produire une parole "qui coule"6670. Même après la mort de ce pauvre homme, le lecteur entend le bruit de son corps en tombant mort par terre:

“ [...] à la fin le seul monde possible c'était l'écho du fracas de son corps $[\ldots]^{\prime, 71}$.

Cette image nous montre que, comme l'affirme Myrthe Holvoet, "ce qui reste est un corps creux qui fait résonner les paroles non-dites de la victime" ${ }^{\text {,72. }}$. Remarquons que Mauvignier donne une importance distinguée à l'écho dans ses romans ; c'est ce qu'il explique, lors d'un entretien, en répondant à la question: "l'écriture [lui] donne-t-elle les moyens de capter cet écho, de saisir et d'exprimer ce que sont les autres ? De saisir leur vérité ?":

"Pas la vérité, ce serait prétentieux de dire ça. L'écriture est un moyen d'appréhension de l'air qui circule entre les êtres, de l'air dont ils sont constitués. Je crois de moins en moins à la profondeur ... Davantage au côté poreux, creux, à la vibration ... L'idée de profondeur me fait trop penser à une essence, une finalité. Quelque chose

${ }^{67}$ Ibid., p. 22

${ }^{68}$ Ibid., p. 25

${ }^{69}$ Sur le sujet du non-dit dans les œuvres de Mauvignier, voir « L'objet indicible et la phrase circulaire de Laurent Mauvignier de Frédéric Martin-Achard, p. 93-112 et «Ce qui le récit ne dit pas: l'événement, une énigme à l'œuvre dans les récits de Laurent Mauvignier de Julie Gresh dans La langue de Laurent Mauvignier: « une langue qui court », op. cit., p. 113-123.

${ }^{70}$ Myrthe Holvoet, op. cit., p. 61.

${ }^{71}$ L. Mauvignier, Cqjo, op. cit., p. 36.

${ }^{72}$ Myrthe Holvoet, op. cit., p. 63. 
comme une vérité établie ... Je ne sais pas ce qu'est le soleil, mais je sais ce qu'est la sensation de sa chaleur sur ma peau. Je me contenterais de l'exploration de cette sensation pour dire quelque chose et de l'être, et du soleil ... C'est juste rendre compte d'une expérience. Le monologue rétablit cette fragilité-là. Il ne part pas d'une certitude" ${ }^{\text {"73. }}$.

D'autre part, au niveau sonore du roman, Mauvignier emploie régulièrement les figures de style, comme, entre autres, l'allitération et l'assonance qui donnent une sonorité au texte au niveau des sensations auditives. La fréquence de ces figures de style crée dans le texte un espace de jeu sur les sonorités. Citons cet exemple:

${ }^{6}[. .$.$] il pourrait dire je vaux, je valais, une vie doit$ valoir un peu plus qu'une bière [... $]^{\text {"9 } 74}$.

L'allitération du son [v] nous donne à penser au mot "vie". D'autres exemples d'assonance se manifestent comme:

"[...] et dans sa tête, l'espoir aussi en miettes, pas maintenant, pas comme ça, et le sang dans les tympans, peut-être, le sang dans la bouche aussi, là où des mots obscurs attendent comme le monde quand il dort sous la neige, [... $]^{\text {"75 }}$.

Dans cet exemple l'alternance de l'assonance du son

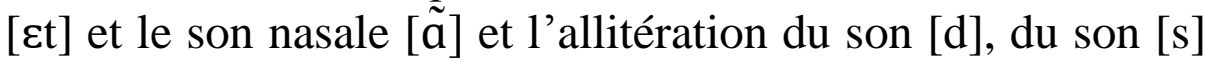
et du son [m] avec la répétition des mots "sang" et "comme" créent un effet très distingué de sonorité. Ajoutons que la comparaison entre "des mots obscurs" et "le monde quand il

${ }^{73} \mathrm{En} \quad$ ligne: http://www.laurent-mauvignier.net/a-l-occasion-de-dans-la-fouleentretien.html, consulté le 03/01/2017.

${ }^{74} \mathrm{~L}$. Mauv ${ }^{74}$ En ligne: http://www.laurent-mauvignier.net/a-l-occasion-de-dans-lafoule-entretien.html, consulté le 03/01/2017.

ignier, Cqjo, op. cit., p. 40.

${ }^{75}$ Ibid., p. 52. 
dort sous la neige" augmente le sentiment de désespoir et d'abattement et annonce le "shutdown" de la parole, pour reprendre les paroles de Myrthe Holvoet.

Prenons un autre extrait:

"[...] mais ça, c'est encore une façon d'espérer un truc, comme au dernier moment, quand il $y$ avait cette voix qui continuait et répétait, pas maintenant, pas comme ça, jusqu'à ce qu'elle se taise elle aussi et s'efface dans un chuchotement, trois fois rien, un sifflement, sa voix à lui qui continuera dans ta tête, à murmurer, à répéter toujours pas maintenant, pas maintenant, pas comme ça, pas maintenant-" ${ }^{76}$.

Dans cet exemple, la répétition régulière du son [s] crée un rythme répétitif et un effet de cadence donnant à entendre les sifflements et les chuchotements de la victime qui est sur le point de mourir. Cependant, l'écho de son agonie et de ses murmures restera à toujours dans la mémoire des gens même après sa mort car si le pauvre homme est écrasé par la violence, il reste la voix qui, luttant contre ce qui l'oppresse, le fait vivre.

Ainsi, comme nous l'avons déjà montré, le vocabulaire qui stimule les sensations auditives chez le lecteur est riche et varié. Il constitue une matière rythmique et acoustique qui donne à entendre tout.

\subsection{L'odorat}

Dans Ce que j'appelle oubli, le sens de l'odorat est lié trop souvent à la violence comme le témoigne l'exemple suivant:

"[...] il fait un mouvement pour retirer son épaule, mais un autre [un des vigiles] prend son bras, les

${ }^{76}$ Ibid., p. 62. 
doigts très écartés, fermement, il sent l'anneau froid et lisse sur son bras nu, un déodorant ou une eau de toilette qu'il connaît et lui rappelle une odeur de poivre [...]" $]^{977}$.

La victime perçoit l'odeur du déodorant d'un des vigiles qui lui rappelle l'odeur du poivre. Le choix de cette odeur de poivre est, à notre avis, particulièrement significatif puisque cette odeur est très forte, épicée, montante, sèche et herbacée. Elle pique le nez. Cette odeur connue par tout lecteur lui permet de deviner la violence intense à venir à cet homme malheureux. L'odeur poivrée du parfum du vigile est répétée à maintes reprises pour insister sur le fait que la victime a perdu toute sa force de résistance aux coups des vigiles et, d'autre part, pour renforcer l'effet de danger et de drame à venir: "[...] il faudrait de la force et il n'en a presque plus, à peine encore pour percevoir cette odeur poivrée du parfum $[\ldots]^{978}$.

La sensation olfactive est associée également au souvenir et au sexe, la victime n'a-t-il pas dit, avec beaucoup d'amertume, que:

"[...] je sais bien que je fais le mort mieux que personne, mais je ne me plains pas parce que, l'amour, je l'ai fait si souvent, je l'ai rencontré si souvent, des visages et des prénoms, des voix et des mains, des odeurs, des parfums et des sexes, alors je ne me plains de rien sauf d'avoir glissé trop vite, si vite, dans la mort [... $]^{\text {"79 }}$.

Ainsi, la perception olfactive joue-t-elle un double rôle: d'une part, elle matérialise la situation grave dans

77 Ibid., p. 13.

${ }^{78}$ Ibid., p. 26.

${ }^{79}$ Ibid., p. 61. 
laquelle la victime se trouve, d'autre part, elle évoque ses souvenirs d'amour pendant sa vie de vagabond.

\subsection{Le goût}

Dans ce roman les termes d'ordre gustatif sont peu nombreux. Il montre souvent l'état d'âme d'une personne sans travail, ni argent:

"[...] il avait juste envie d'une bière, tu sais ce que c'est l'envie d'une bière, il voulait rafraîchir sa gorge et enlever ce goût de poussière qu'elle avait et qui ne le lâchait pas, [...] $)^{80}$.

Dans cet exemple, le narrateur explique que c'est la soif torturante qui a poussé le jeune homme à prendre la canette et la boire sans avoir de l'argent pour la payer. L'expression en italique nous fait mieux comprendre l'état psychologique et moral du jeune homme qui souffre de problèmes sociaux.

Citons un autre exemple où la victime explique que sa mort n'est pas, selon lui, l'événement le plus triste du monde, mais ce qu'il importe, c'est l'injustice de sa mort et l'arrêt d'une vie pleine d'activité et d'émotions pour le simple plaisir personnel des quatre assassins:

“[...] il dirait ma mort n'est pas l'événement le plus triste de ma vie, ce qui est triste dans ma vie c'est ce monde avec des vigiles et des gens qui s'ignorent dans des vies mortes comme cette pâleur, cette mort tout le temps, tous les jours, que ça s'arrête enfin, je t'assure, ce n'est pas triste comme de perdre le goût du vin et de la bière, le goût d'embrasser, d'inventer des destins à des gens dans le métro et le goût de marcher des heures et des tas de choses que

${ }^{80}$ Ibid., p. 8. 
je ne ferai jamais, que je n'aurais jamais faites de toute façon $[\ldots]$ "..

Ici, le mot "goût", repris pour trois fois, stimule chez le lecteur des sensations de différents niveaux: le niveau dégustatif "le goût du vin", le niveau passionnel et charnel "le goût d'embrasser", et enfin le niveau physique et actionnel "le goût de marcher" indiquant ainsi la passion de la victime de se balader dans les rues pendant des heures et des heures sans se fatiguer.

Nous distinguons également une certaine correspondance entre le visuel et le gustatif, comme dans l'exemple suivant:

"[...] tu te rappelles ? les vacances, Noirmoutier, les premières filles aux seins nus que vous suiviez sous les pins et tous les souvenirs qui font remonter des bouffées de couleurs, le bleu du ciel, le gris de l'eau et l'eau salée sur les lèvres, tu t'en rappelles ? $[\ldots]^{982}$.

Ainsi, au delà d'exprimer une expérience gustative particulière, le vocabulaire du goût indique, d'une part, les problèmes sociaux et psychologiques dont souffre le jeune homme marginal et, d'autre part, il prend une dimension passionnelle et charnelle.

\subsection{Le thermique}

Les sensations relatives au froid sont nombreuses et, le plus souvent, associées à la mort et à la violence:

"[...] mais un autre [un des vigiles] prend son bras, les doigts très écartés, fermement, il sent l'anneau froid et lisse sur son bras nu $[\ldots]^{\prime \prime 83}$.

${ }^{81}$ Ibid., p. 60.

${ }^{82}$ Ibid., p. 57.

${ }^{83}$ Ibid., p. 13. 
L'expression d'"anneau froid" est évocatrice. D'une part, le mot "anneau" forme un cercle de métal comme le cercle que les quatre vigiles forment autour du jeune homme pour le punir et le battre à mort, d'autre part la sensation de la froidure de l'anneau du vigile sur le bras de la victime est métaphorique dans la mesure où le choix de l'adjectif "froid" pour qualifier "l'anneau" nous fait penser à la violence du processus de mise à mort de la victime que les vigiles décident froidement d'exécuter en s'amusant et à "cœur joie".

La froidure est évoquée plusieurs fois dans ce roman dont nous citons à titre d'exemple:

" [...] il n'avait pas encore en tête la violence des coups à venir et le froid de la dalle de ciment, car, au début, il ne peut pas se douter ni imaginer qu'il ne lui restera bientôt que la nudité et la froidure sur un matelas de fer ou d'inox, et aussi, attachée à un doigt de pied, une étiquette avec son nom, un numéro, [... $]^{\text {\$, } 84}$.

La froidure met en évidence la cruauté de la mise à mort de la victime et l'état de son corps mort étendu sur le béton du sol "froid" avec ses "blessures froides" $" 85$, et posé ensuite sur un matelas de fer ou d'inox "froid" dans un frigo "froid".

$\mathrm{Si}$, dans ce roman, la sensation de froid est souvent associée à la mort, la sensation de chaleur a des rapports avec le meurtre dont nous repérons les occurrences suivantes:

"[...], c'est si lourd sur leurs bras et dans leurs jambes, avec cette bouillie d'idées dans leur tête

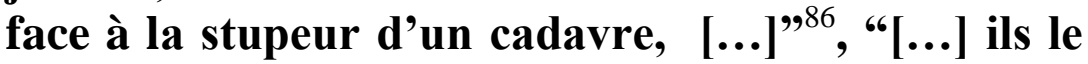

\footnotetext{
${ }^{84}$ Ibid., p. 17.

${ }^{85}$ Ibid., p. 18.

${ }^{86}$ Ibid., p. 27.
} 
frappent, tout ce qui leur a fait du mal dans la vie, c'est comme ça qu'ils font pour continuer à s'échauffer, en se racontant des histoires, [...]" ${ }^{\text {"87 }}$.

Les vigiles, en frappant fort le pauvre homme, veulent se venger de tout le mal dont ils ont souffert dans leur vie et cette pensée les réchauffe et les fait continuer à frapper la victime jusqu'à la mort. Le chaud est donc lié à l'idée de la vengeance.

Ainsi l'auteur emploie-t-il le vocabulaire thermique relatif au froid et à la chaleur pour exprimer la cruauté de la violence et de la mort. Notons que le narrateur adresse sa parole au frère de la victime en tant que son interlocuteur, ce dernier n'échange pourtant jamais la parole avec le narrateur. Cependant, cet interlocuteur muet constitue un élément générateur dans le roman dans la mesure où le narrateur ne s'adresse pas seulement au frère de la victime, mais il s'adresse à nous, lecteurs, nous "frères humains" reprendre les termes d'Albert Cohen, qui avons froid en lisant ce drame. Le froid qui domine dans ce roman prend donc une toute autre ampleur en introduisant une dimension politique et humaine à ce drame en vue de nous inciter à refuser ce monde où la mort devient banale et régulière, et nous l'acceptons simplement sans dire un mot.

\subsection{Le tactile}

Le vocabulaire du toucher est pauvre dans ce roman. Il a des rapports avec la psychologie de la victime comme le montre l'exemple suivant:

"[...] il est entré dans le supermarché sans l'intention de rien sinon marcher entre les rayons et laisser errer ses yeux comme il faisait parfois, à Montparnasse ou ailleurs, sur les tombes des gens

${ }^{87}$ Loc.cit.

${ }^{88}$ Albert Cohen, Ô vous, frères humains, Gallimard, Paris, 1972. 
célèbres et où les noms dorés sur les marbres sont comme des labels de qualité, et dans les rayons il a aimé toucher les papiers, les emballages, le plastique, la cellophane, mais il ne prenait jamais rien et il a dû seulement avoir si soif soudain, voilà, il n'a pas pu résister et son doigt a dégoupillé la canette $[. .$.$] ]" 89$.

Le jeune homme aime seulement sentir la matérialité des objets par ses mains, sans néanmoins désirer les posséder. Cela doit peut-être aux difficultés sociales qu'il subit en tant qu'homme marginal menant une vie de clochard.

Par ailleurs, un sens figuré du toucher se manifeste lors de la description de la victime qui était en train de mourir:

"[...] quand c'était au bord de la fin, touchant déjà la fin, y glissant [...]"

Il s'agit d'une concrétisation de la mort de laquelle le jeune homme s'approche. Notons que, même en mourant, la victime avait l'espoir que les vigiles allaient s'arrêter de frapper et sa vie allait tenir.

Le sens du toucher prend donc deux dimensions: une psychologique qui montre la passion de la victime de sentir la matérialité des objets par ses mains, ce qui désigne peutêtre des problèmes psychiques et sociaux dont souffre ce jeune marginal et une autre poétique qui exprime un sens figuré du toucher concrétisant quelques vérités abstraites comme la mort.

\subsection{Les matières}

Étonnante est la fréquence de quelques référents privilégiés qui reviennent régulièrement au fur et à mesure de

${ }^{89}$ L. Mauvignier, Cqjo, op. cit., p. 51-52.

${ }^{90}$ Ibid., p. 48.

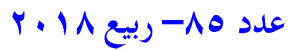


la lecture. Ils donnent une spécificité au dispositif du roman au niveau textuel, mais aussi au niveau idéologique. Le métal constitue un référent que nous rencontrons trop souvent dans ce texte sous plusieurs formes dont les plus fréquentes sont "la lame de couteau"", "les lames aiguisés""92, mais également "la canette de bière" ${ }^{93}$. Les équipements dans le supermarché comme "les caddies", "les palettes" et "les chariots élévateurs" sont en métal. Des référents métalliques apparaissent encore comme par exemple "l'osier qui craque"," "les montagnes de bouffe et de fer""," "le matelas de fer et d'inox" $" 96$, "l'étal du père" $"$ " Nous repérons, de même, une métaphore qui évoque le métal: "le son métallique de chanson à la radio" ${ }^{98}$. Ce son qui fait un écho et un bruit de métal est également significatif dans ce point de vue.

Les objets en métal figurent, avant d'être les référents du discours, la matérialité de notre vie contemporaine marquée par la brutalité et la violence quotidiennes où le côté moral et éthique est de plus en plus diminué. L'emploi excessif de ces référents joue un rôle très important dans le dispositif idéologique de ce roman dénonçant la violence de certains gens dans nos sociétés qui causent quotidiennement la mort d'autrui. La portée idéologique de ce roman se présente comme une: "métaphore à peine cachée de l'affrontement inique entre capitalisme et pauvreté, Ce que j'appelle oubli se lit comme la tragédie d'une société

\footnotetext{
${ }^{91}$ Ibid., p. 9, 40, 42.

92 Ibid., p. 45.

${ }^{93}$ Ibid., p. 7, 12, 15,16, 40, 52.

${ }^{94}$ Ibid., p. 56.

95 Ibid., p. 41.

${ }^{96}$ Ibid., p. 17.

${ }^{97}$ Ibid., p. 44

${ }^{98}$ Ibid., p. 11.
} 
aveugle où l'homme seul face à la machine, victime anonyme écrasée par un système qui a cessé d'être humain, est dépossédé à sa mort de la peur de manquer, de la peur d'avoir soif. Mais cette société est la nôtre, ce que Laurent Mauvignier n'omet pas de nous rappeler"99.

À côté du métal, le plastique constitue également un élément que l'auteur emploie régulièrement dans le dispositif textuel du roman: "cheval en plastique" "100, "pelouse synthétique" "101, "grandes portes en plastique transparents" "102, "le film plastique"103, "un plastique" $"$ " Cela insiste sur l'artificialité de la vie moderne qui empêche les gens de prendre conscience de la vraie vie des autres en la méprisant ou l'ignorant. Le narrateur de Ce que j'appelle oubli fait parler la mort pour nous transmettre un message particulier qui nous touche profondément: “ma mort n'est pas l'événement le plus triste de ma vie, ce qui est triste dans ma vie c'est ce monde avec des vigiles et des gens qui s'ignorent dans des vies mortes comme cette pâleur"105. Cette voix qui résonne dans le vide fait advenir, comme l'espérait Mauvignier "quelque chose de notre écoute, dans le double sens du mot: écouter, et être à l'écoute. S'ouvrir, esthétiquement, politiquement, à quelque chose de la fraternité" 106 .

99 En ligne https://rhinoceros.eu/2011/03/ce-que-j-appelle-oubli-de-laurent-mauvignier/ 3 mars 2011, consulté le 10/06/2017.

100 L. Mauvignier, Cqjo, op. cit., p. 10.

${ }^{101}$ Loc. Cit.

102 Ibid., p. 20.

${ }^{103}$ Loc. cit.

104 Ibid., p. 18.

105 Ibid., p. 60.

${ }^{106}$ En ligne http://www.chateauvallon.com/IMG/pdf/19. ce que j_appelle_oubli.pdf, consulté le 14/03/2017. 


\section{"Le dalle de béton"107 un dernier élément qui se} répète régulièrement, est souvent associé au sang, au froid et à la mort. L'emploi des matières met donc en évidence la portée idéologique du roman qui dénonce l'absurdité de la vie et la lâcheté des hommes avec l'espoir de vaincre les sentiments d'échec, de déception généralisée, d'impuissance lâche des gens dans les sociétés contemporaines.

\section{Conclusion}

L'écriture de Ce que j'appelle oubli est une écriture particulière qui donne à voir, à entendre et à ressentir. Elle se distingue par un caractère évocateur qui présente des expériences visuelles et sensorielles entremêlant la vue, l'ouïe, l'odeur, le goût et le toucher, ce qui met en évidence l'aspect multisensoriel de l'œuvre. Elle crée ainsi une composition synesthésique qui se rapproche de la conception de la plasticité actuelle comprise comme "l'ensemble des dispositifs artistiques donnant à voir et à ressentir la représentation" ${ }^{108}$. Cet aspect multisensoriel inscrit le langage de Mauvignier dans une esthétique de perception et de sensation où les personnages projettent leur tension interne en la faisant voir au lecteur pour qu'il puisse ressentir et revivre avec eux leur drame.

Les sensations visuelles constituent un élément majeur dans la matérialité textuelle de ce roman. Elles présentent un tableau riche de stylèmes relatifs à la vue que ce soient des verbes, des noms ou bien des adjectifs, avec beaucoup de lumière et de couleur. Ce qui est, en effet, le plus fascinant dans ce roman, c'est la façon de montrer visuellement ce qui se passe au lieu de tout dire. Par ailleurs, l'action de voir et d'entendre établit une relation d'équivalence où la vue stimule

${ }^{107}$ L. Mauvignier, Cqjo, op. cit., p. 22, 23, 25, 47, 52.

${ }^{108}$ Christophe Henry, Art des temps modernes et Historiographie, Univ. de Lyon II, 2007-2008 (en particulier le paragraphe sur Le sujet et la plasticité), p. 128. 
constamment l'ouïe dans une activité réciproque interminable. Elles fonctionnent ensemble et se complètent.

Quant aux sensations non-visuelles, elles jouent également un rôle important dans ce roman dans la mesure où elles créent un ton affectif qui submerge le lecteur et lui fait revivre les événements tragiques du roman. Les sensations nonvisuelles se divisent en quatre genres correspondant aux quatre sens suivants: l'ouie, l'odorat, le goût et le toucher.

Le vocabulaire qui stimule les sensations auditives chez le lecteur est riche. Il constitue une matière rythmique et acoustique qui donne à entendre. Quant à la perception olfactive, elle joue un double rôle. Elle matérialise, d'une part, la situation grave dans laquelle la victime se trouve et, d'autre part, elle évoque ses souvenirs d'amour pendant sa vie de vagabond. Par ailleurs, au delà d'exprimer une expérience gustative particulière, le vocabulaire du goût indique, d'un côté, les problèmes sociaux et psychologiques dont souffre la victime et il prend, de l'autre, une dimension passionnelle et charnelle.

Le vocabulaire thermique relatif au froid et à la chaleur met en relief la cruauté de la violence et de la mort. Quant au sens du toucher, il concrétise quelques vérités abstraites comme la mort en la personnifiant. La dimension idéologique du roman, dénonçant la violence et la brutalité quotidiennes de la vie où le côté moral et éthique est de plus en plus diminué, est mise en exergue grâce à un emploi récurrent de certains objets concrets issus des métaux comme le fer et l'inox. D'autre part, cet emploi crée un effet d'art plastique et de composition synthétique dans le texte.

Ainsi, le pouvoir évocateur puissant du dispositif textuel particulier du roman éveille-t-il la sensibilité du lecteur dès les premières lignes. Ce pouvoir permet certainement de cerner la littérarité de ce roman et le ranger 
parmi les œuvres de grande valeur dans la production littéraire postmoderne. C'est comme l'affirme fort justement P. Muriel: "Lire Mauvignier, c'est ainsi d'abord entrer dans un univers stylistique qui dépasse le seuil de l'histoire. Chez lui, le signifiant précède et organise la pensée au fondement du récit. Les particularités stylistiques de ses romans nous immergent en effet dans une écriture où l'on "subit" une violence faite au verbe, originalité qui préfigure la configuration mentale bouleversée des personnages. $L$ 'aspect à la fois sonore et mouvant de cette langue saturée de significations dans son seul versant formel donne à éprouver un sentiment ou une sensation indépendamment de la connaissance référentielle et sémantique du texte" ${ }^{\text {"109. }}$.

Références

I. Corpus

- MAUVIGNIER Laurent, Ce que j'appelle oubli, Éditions de Minuit, 2011.

\section{Ouvrages et articles consacrés à Laurent}

Mauvignier

- BONAZZI Matilde, “'’Ce qu'on pourrait appeler un style": les représentations de la pratique stylistique de Laurent Mauvignier dans la critique littéraire", la langue de Laurent Mauvignier "une langue qui court", Jacques Dürrenmatt et Cécile Narjoux (dir.), Collection Langages, Éds. Universitaires de Dijon, 2012.

- FAYETTE Yann, GONZALEZ Remi et MATEO Anaïs, "Mauvignier: impressions", La Langue de Mauvignier: "une langue qui court", Jacques

${ }^{109}$ Pfefferlé Muriel, Autour de quatre romans de Laurent Mauvignier: le déploiement d'une « écriture du subi », Mémoire de Maîtrise universitaire, Sous la direction du Professeur Jean Kaempfer, Université de Lausanne, 2011, p. 98. 
Dürrenmatt et Cécile Narjoux (dir.),

Collection Langages, Éds. Universitaires de Dijon, 2012.

- GERMONI Karine, "L'ironie façon Mauvignier", Existe-t-il un style Minuit ?, M. Bertrand, K. Germoni, A. Jauer (éd), Aix-en-Provence, PUP, 2014.

- GRESH Julie "Ce qui le récit ne dit pas: l'évènement, une énigme à l'œuvre dans les récits de Laurent Mauvignier", La langue de Laurent Mauvignier: "une langue qui court", Jacques Dürrenmatt et Cécile Narjoux (dir.), Collection Langages, Éds. Universitaires de Dijon, 2012.

- GUSTAFSSON Harriet, L'œuvre Romanesque de Laurent Mauvignier, Mémoire sous la direction du professeur Björn Larsson, Université de Lund Centre de langues et de littérature, 2012, en ligne: lup.lub.lu.se/student-

papers/record/3812729/file/3812804.pdf, consulté le $29 / 03 / 2017$.

- HOLVOET Myrthe, Laurent Mauvignier et le minimalisme: l'écriture du non-dit, Mémoire de maîtrise sous la direction du prof. Pierre Schoentjes, Faculté de Philosophie et Lettres, Université Gent, 2012-2013.

- LAFERRIERE Aude, "Entre "le silence et l'écho", les réticences et les réminiscences verbales: L'écriture des discours rapportés dans Loin d'eux et Des hommes", La langue de Laurent Mauvignier "une langue qui court", Jacques Dürrenmatt et Cécile Narjoux (dir.), Collection Langages, Éds. Universitaires de Dijon, 
2012.

- LEBRUN Jean-Claude, "Laurent Mauvignier, une insurmontable solitude”, L'Humanité, 21/09/2000, en ligne: www.humanite.fr, consulté le 12/04/2017.

- MARTIN-ACHARD Frédéric, "L'objet indicible et la phrase circulaire de Laurent Mauvignier", La langue de Laurent Mauvignier: "une langue qui court", Jacques Dürrenmatt et Cécile Narjoux (dir.), Collection Langages, Éds. Universitaires de Dijon, 2012.

- MURIEL Pfefferlé, Autour de quatre romans de Laurent Mauvignier: le déploiement d'une "écriture du subi", Mémoire de Maîtrise universitaire sous la direction du Professeur Jean Kaempfer, Université de Lausanne, 2011.

- SAUVAGE Christian, "Le miracle Mauvignier", Le Journal du Dimanche, 3 avril 2005, en ligne: http://leseditionsminuit.fr, consulté le 15/04/2017.

III. Entretiens avec Laurent Mauvignier

- DUBOR Françoise, "Entretien avec Laurent Mauvignier autour de Dans la foule (2006)". Cahiers Forell, 2015. En ligne: http://09.edel.univpoitiers.fr/lescahiersforell/index.php?id=251, consulté le 27/07/2017.

- FAERBER Johan, Entretiens, Laurent

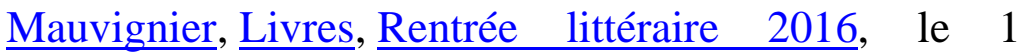
septembre 2016, en ligne: https://diacritik.com/2016/09/01/laurent-mauvignier-ily-a-des-livres-qui-veulent-nous-soumettre-a-nos-peursplutot-que-de-les-interroger/, consulté le 17/12/2017.

- "Capter la surface des choses", Entretien avec Jean Laurenti, Le Matricule des anges, Octobre 2006, 
en ligne: http://www.laurent-mauvignier.net/entretiens/dans-la-foule/capter-la-surface-deschoses.html, consulté le 05/07/2017.

- "Misère de l'amour. Entretien avec Jean Laurenti", Le Matricule des Anges, mars 2004, en ligne: http://www.laurentmauvignier.net/entretiens/seuls/misere-de-1amour.html, consulté le $24 / 05 / 2017$.

IV. Ouvrages et articles généraux

- BRICCO Elisa, 'Notes à partir de L'esthétique de la littérature entre performativité et visualité de $\mathrm{M}$. Fusillo", ARGEC, 15-6-2012, en ligne, http://argec.hypotheses.org/243, consulté le 10/07/2017.

- CLERC Jeanne-Marie, Littérature et cinéma, Coll. Fac. Cinéma, Nathan Université, 1999.

- COHEN Albert, Ô vous, frères humains, Gallimard, Paris, 1972.

- HENRY Christophe, Art des temps modernes et Historiographie, Université de Lyon II, 2007-2008.

- LEUTRAT Jean-Louis (dir.), Cinéma et littératurele grand jeu, De l'incidence (éditeur), 2010.

- MOLINIE Georges, La stylistique, PUF, 2001.

V. Site internet

- http://www.laurent-mauvignier.net/a-l-occasion-dedans-la-foule-entretien.html, consulté le 03/01/2017.

- $\quad$ https://rhinoceros.eu/2011/03/ce-que-j-appelleoubli-de-laurent-mauvignier/, consulté le 10/06/2017.

- http://www.chateauvallon.com/IMG/pdf/19._ce_qu e_j_appelle_oubli.pdfconsulté le 14/03/2017. 
الظواهر الدلالية في كتاب إعراب القراءات والشواذ "لأبي البقاء العكبري"

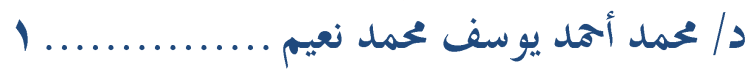

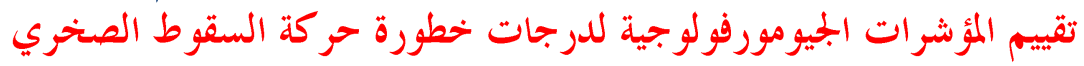

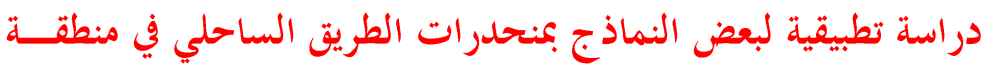

$$
\begin{aligned}
& \text { أ.د/ مني عبد الرحن يس الكيالي }
\end{aligned}
$$

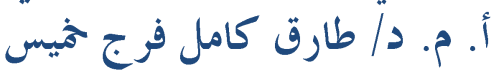

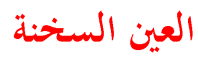

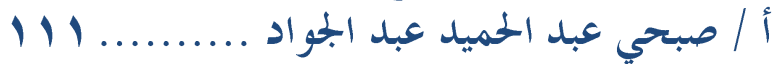

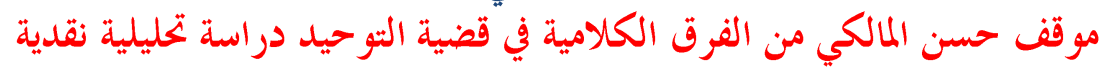
د/ الطالبة/ شريفة أحد المالكي .................

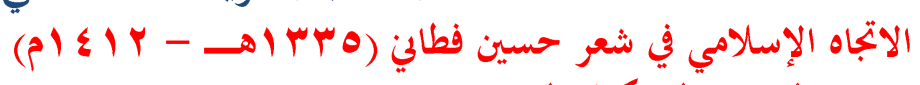

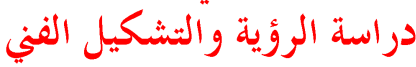

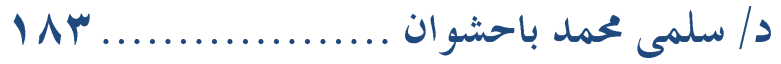

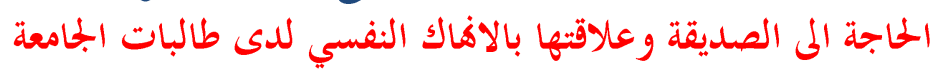

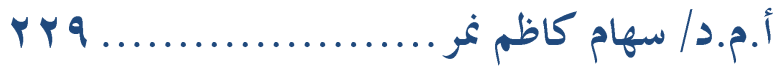

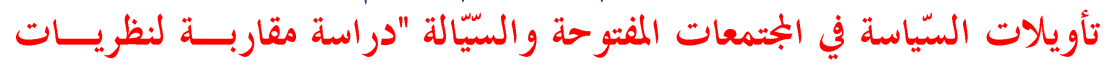

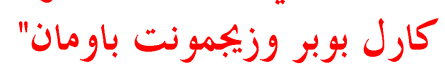

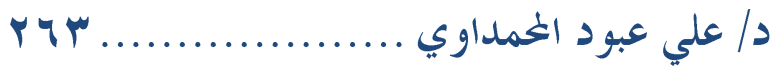

الأفعال الإنــــــــية في الأربعين النووية "دراسة تداولية" ديملية

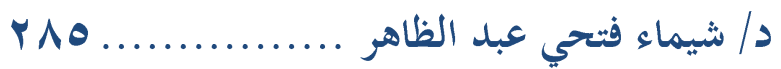

Title: Lexical Organization: "Sound Emission" Verbs

Dr. Salih Alzahrani..............................1

INTONATION GROUPS IN ARABIC (MAINLY IN

THE HOLY QURAN)

Dr. Areej Ali Otay.....

Voir, entendre et ressentir: à propos de l'écriture de Ce que j'appelle oubli de Laurent Mauvignier

Dr. Dalia Metawe 
من أعلى الضغوط النفسية والذي يؤثر على علاقات الفرد المختلفة وعلى تفاعلاته مـــع زملائسـهـ وأصدقائه والحاجة إلى صديقة بالنسبة لفتيات الجامعة.

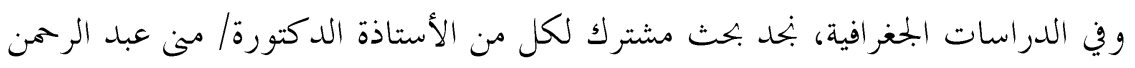

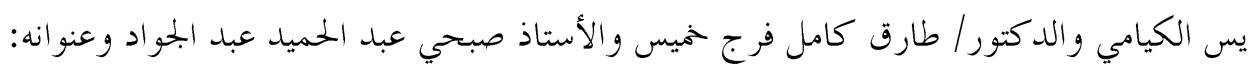

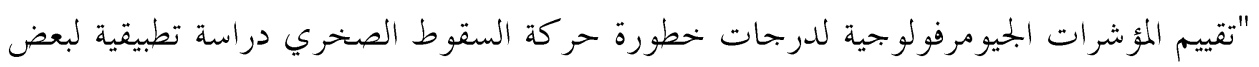

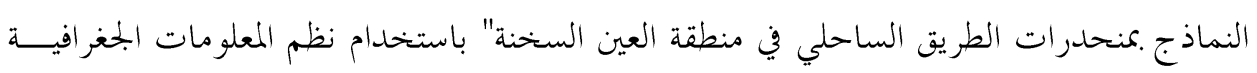

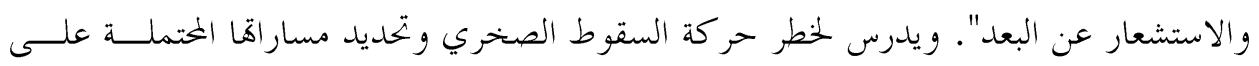

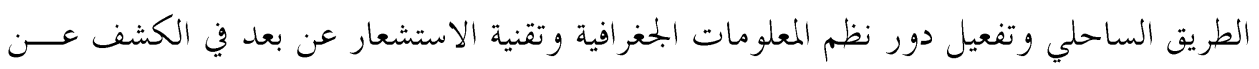
طبيعة منحدر ات تلك المنطقة.

و في مجال الإعلام يأتي بحث الدكتورة/ شيماء فتحي عبد الصادق تحــتـت عنـــوان: "دور

المسرح في علاج ذوي الاحتياجات الخاصة (مسرحية لسه متسماش لعاطف أبو شهبة) نموذجهـاً

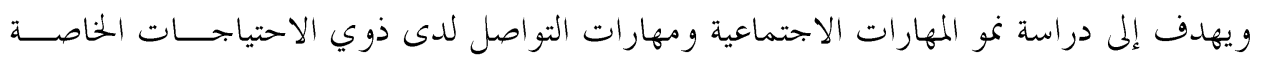

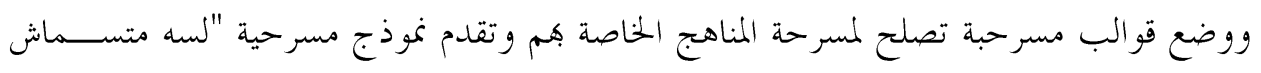
لعاطف أبو شهبة".

نائب رئيس بحلس الإدارة

$$
\text { أ.د / هناء زكريا }
$$

وكيل الكلية للدراسات العليا والبحوث 


\section{افتتاحية العلدد}

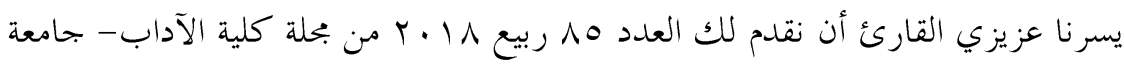

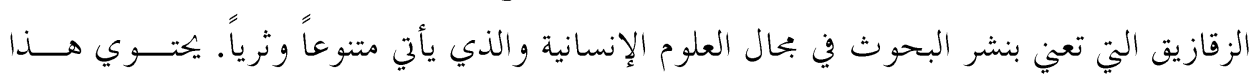

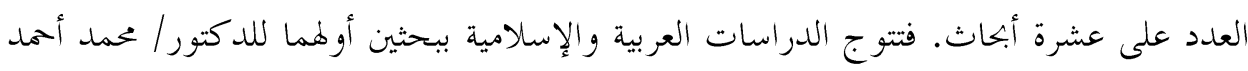

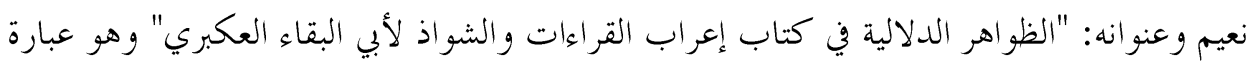

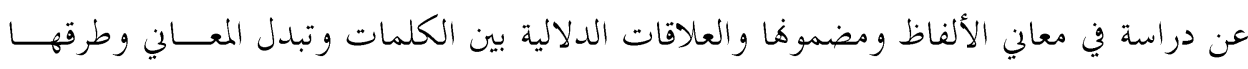

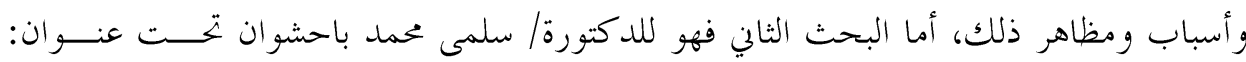

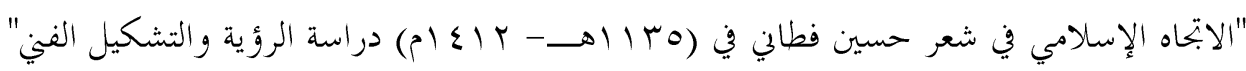
ويتناول الاتحاه الإسلامي في شعر حسين فطاني ودراسة في الرؤية والتشكيل لهذا الشاعر المكيّي.

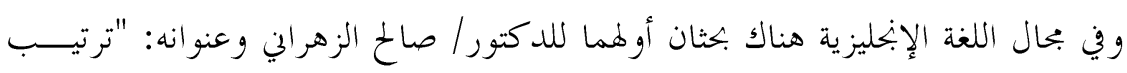

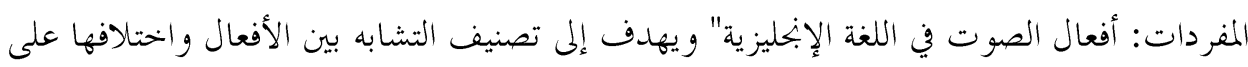

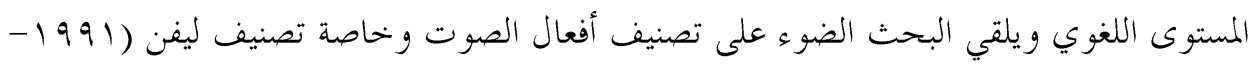

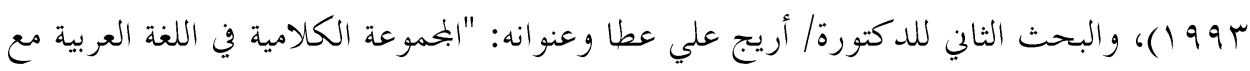

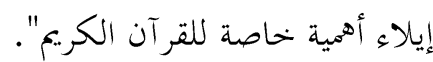

ويأتي بحث اللغة الفرنسية تحت عنوان: "نرى، نسمع، ونشعر فيما يتعلق بالكتابة في رواية

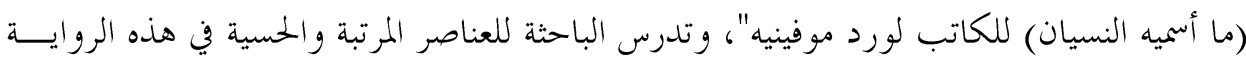
مع تحليل دورها في التنظيم النصي وتركيز الكاتب على العناصر البصرية والحسية وتعدد الحواس.

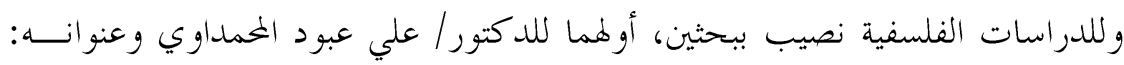

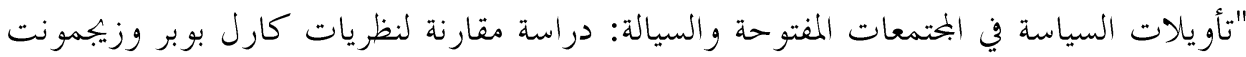

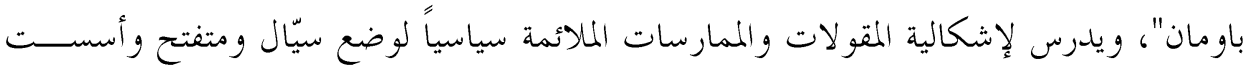

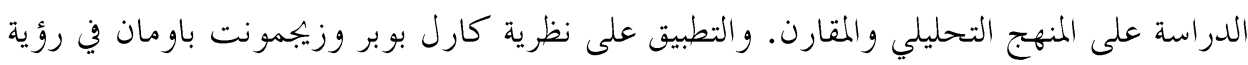

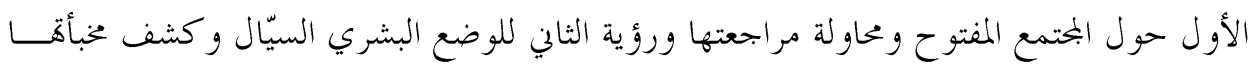

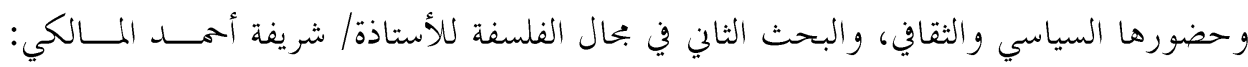

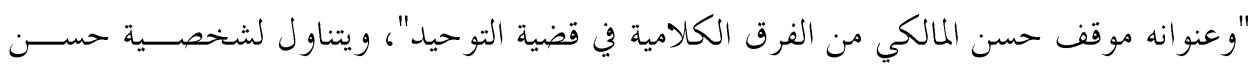

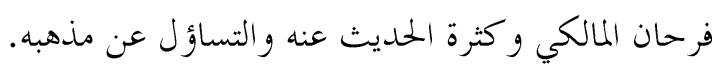
أما علم النفس فيتوّج ببحث للدكتورة/ سهام كاظم نمر وعنو انه: "الحاجة إلى صــديقة وعلاقتها بالإهاك النفسي لدى طالبات الجحامعة" والذي يهدف إلى دراسة الإناك النفسي الذي يعد مركد 

أسماء السادة الأساتذة همكمي هذا العدد ونقا للترتيب الأبجدي

أ.د/ أحمد سالم صالح

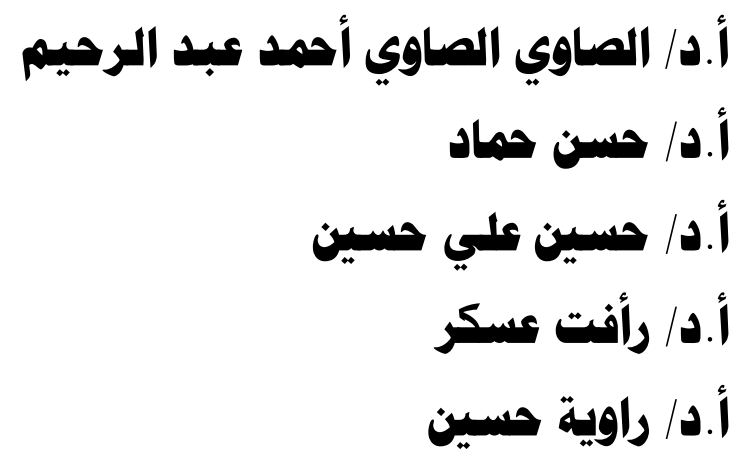
أ.د/ صابر عبد الدايتم يوسف رايه أ.د أد طارق زكريا علي

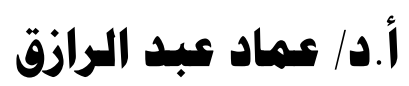
أ.د / لبنى عبد التواب يوسف

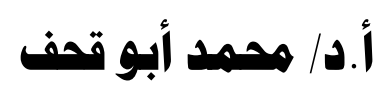

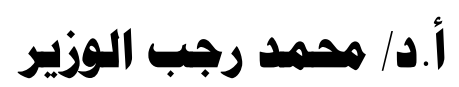

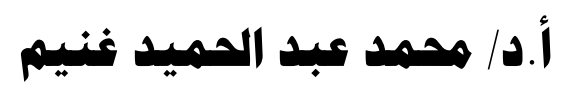
أ.د / هنى أحمد عبد العريز أ.د/ نادية أندراوس أهسي أ.د/ نازك همهد عبد اللطيف الديف أ.د/ نهوى عندس 



\section{هملة كلية \\ هبلة كلية الآداب - جامعة الزتقازيق \\ صدر العدد الأول 1917-17م له}

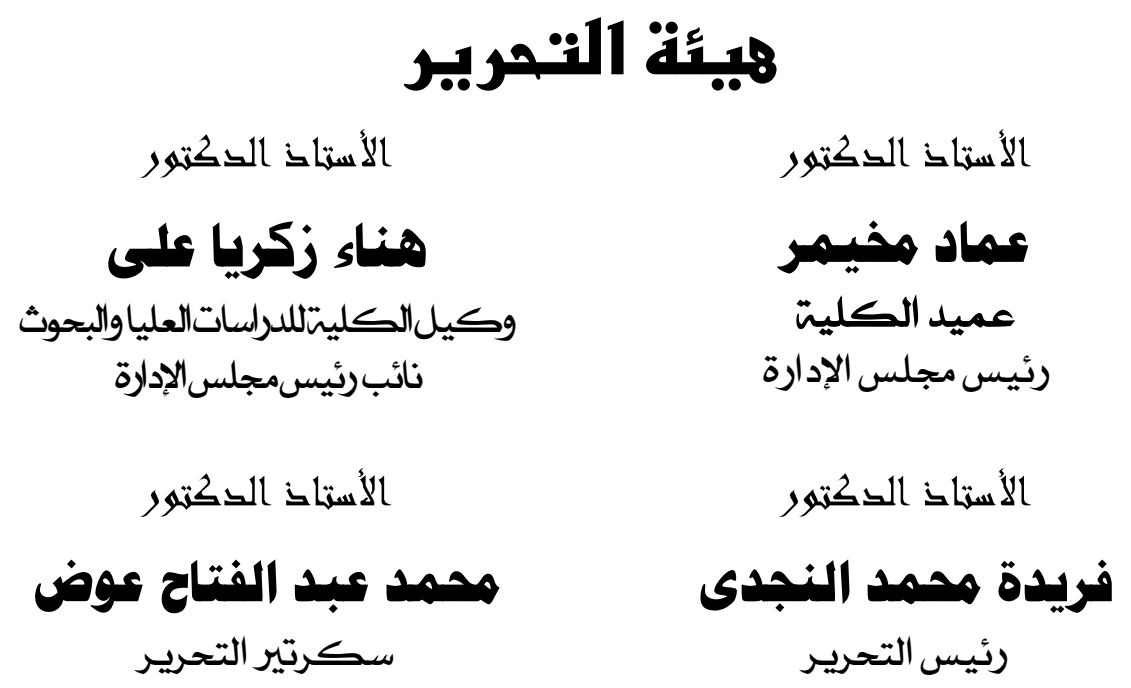

مستثنارو التحريز

أ.د . أحمــــد صـــلاح الـــدين

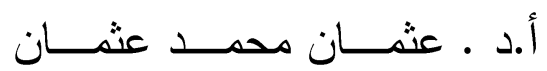

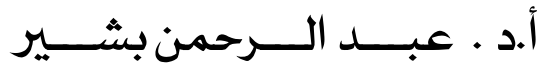

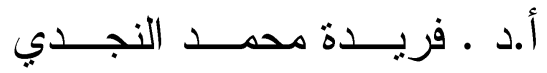

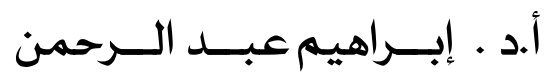

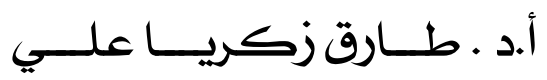

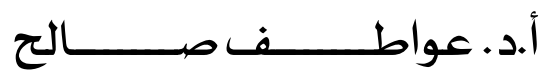

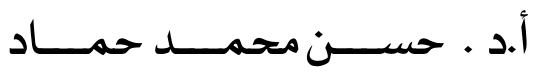

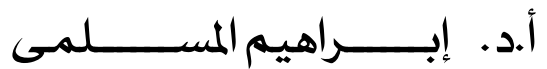


r ا - يرفق ملخصان للبحث باللغتين العربية والإنجليزية على ألا يتجاوز حجم الملخص صفحة واحدة.

با - تتشر المجلة ملخصات الرسائل العلمية العربية والأجنبية. ع ا- تنشر المجلة بحوث معاوني هيئة التدريس كمنطلب للحصول علـى درجني الماجسنير و الاكتور اه.

10 - تتشر المجلة بحوث أعضاء هيئة التدريس بدرجة أستاذ وفق القيمــة الفعلية للطباعة. 1 ا 1 - توجه جميع المكاتبات أو الاستفسار ات الخاصة بالنشر إلــى رئسبس تحرير المجلة على العنوان التالي.

\section{كلية الآداب - جامعة الزتقان النقيق}

تليفون :

http://www.Arts@Zu.edu.eg 
مجلة الكلية الآداب: فصلية- علمية- محكمة تعني بنشر الأبحاث العلمية في مجالات الدر اسة الإنسانية اللغوية و الأدبية و التاريخية و الجغر افية و الفلســفية الإدية

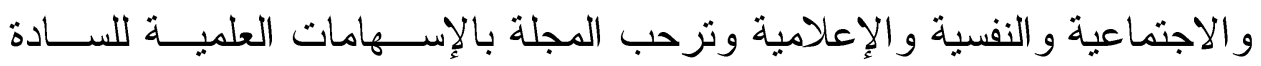

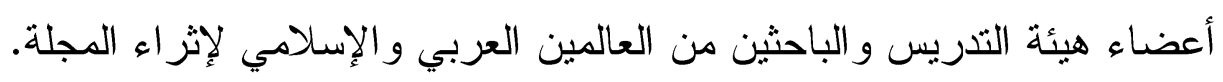

\section{قواعد النشئسر:-}

1- تقبل المجلة البحوث باللغات العربية والإنجليزية و الفرنسية.

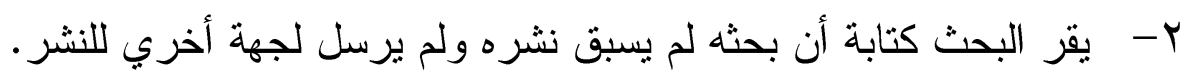

r- يخطر الباحث بخطاب رسمي بقبول النشر في حالة إجازة البحث للنشر .

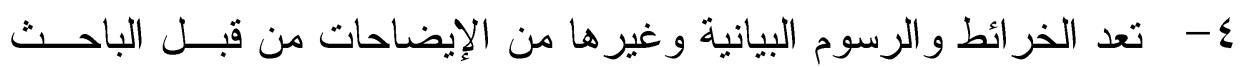

بطريقة تجعلها قابلة للطبع.

- - تعبر البحوث المنشورة عن رأي اصحابها فقط.

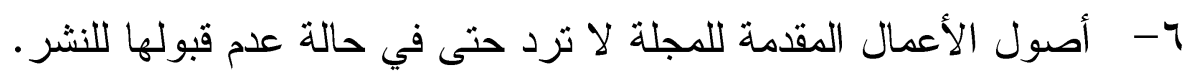

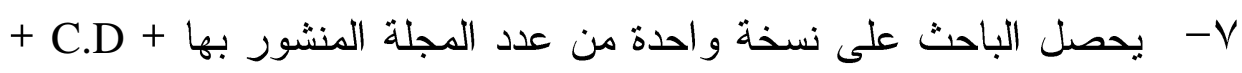
عشر مستلات من البحث.

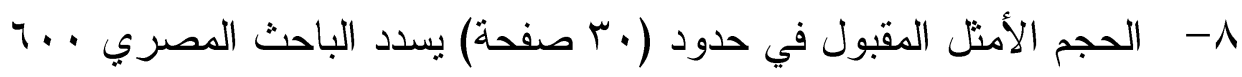

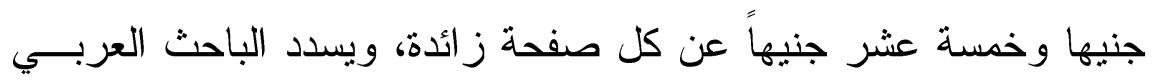
و الأجنبي ... ب دو لار وثثاثة دو لار عن كل صفحة زائدة. 9- يسلم البحث مطبوعاً من أصل وصورتين + C.D على أن يكون مجموعاً

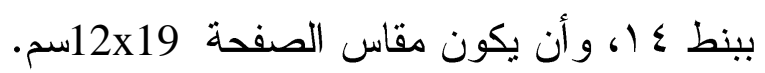

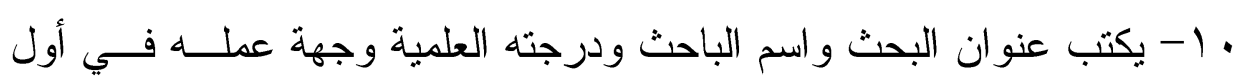
صفحة من البحث. 11 - تكتب المراجع و الهو امش في نهاية البحث، مع الالتز ام بالأسس العلميــة للتوثيق. 



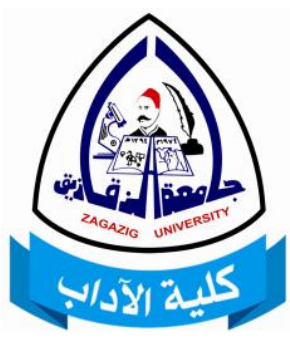

$$
\text { كلية الآداب مجلة }
$$

هملة علمية همكمة فمالية

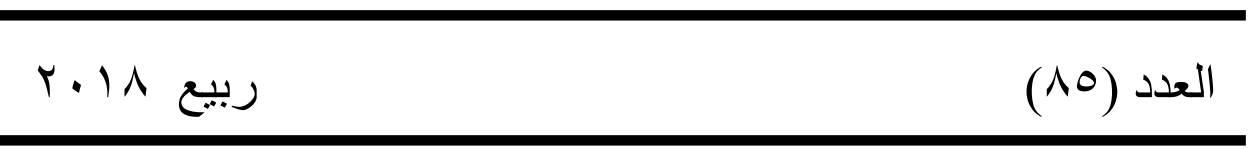

